\title{
Norois
}

Environnement, aménagement, société

$203 \mid 2007 / 2$

Mobilité, hydrologie, loisir et tourisme, espace urbain

\section{L'aménagement des sites de plongée sous-marine en France : une gestion environnementale publique et catégorielle du domaine public maritime?}

The organization of scuba diving sites in France: a public and sectoral environmental management of the maritime public domain?

\section{Olivier Musard}

\section{(2) OpenEdition}

Journals

Édition électronique

URL : http://journals.openedition.org/norois/1526

DOI : $10.4000 /$ norois. 1526

ISBN : 978-2-7535-1551-2

ISSN : 1760-8546

Éditeur

Presses universitaires de Rennes

Édition imprimée

Date de publication : 1 juin 2007

Pagination : $37-50$

ISBN : 978-2-7535-0511-7

ISSN : 0029-182X

Référence électronique

Olivier Musard, «L'aménagement des sites de plongée sous-marine en France : une gestion environnementale publique et catégorielle du domaine public maritime? », Norois [En ligne], 203 | 2007/2, mis en ligne le 01 juin 2009, consulté le 10 décembre 2020. URL : http:// journals.openedition.org/norois/1526 ; DOI : https://doi.org/10.4000/norois.1526 


\title{
L'aménagement des Sites de Plongée sous-marine en France : UNE GESTION ENVIRONNEMENTALE PUBLIQUE ET CATÉGORIELLE DU DOMAINE PUBLIC MARITIME?
}

\author{
OLIVIER Musard \\ Telemme - UMR 6570 CNRS \\ (Université de Provence), \\ Maison méditerranéenne des Sciences de l'Homme, \\ rue du Château-de-l'Horloge, BP 647 - 13064 Aix-En-ProvenCE cedex 2 \\ Conservatoire-Études des Écosystèmes de Provence, \\ 890 chemin de Bouenhoure-Haut \\ 13090 Aix-En-Provence \\ olivier.mus-ean@libertysurf.fr
}

\section{RÉSUMÉ}

L'installation de bouées d'amarrage réduit considérablement l'impact de la pratique de la plongée sous-marine sur les fonds marins. Cette politique d'équipement des sites de plongée intéresse les plongeurs, les opérateurs de plongée, les gestionnaires d'aires marines protégées et dorénavant, des collectivités et des associations. Au regard des autres activités, telles la plaisance et la pêche, aménager des sites de plongée pourrait relever d'une problématique sectorielle et catégorielle. Cette question conduit in fine à s'interroger plus globalement sur le devenir du Domaine Public Maritime et sa gestion, entre logique de publicisation et dynamique de privatisation des territoires littoraux et marins.

Mots CLÉ : Aires marines protégées - Bien public - GIZC - Plongée sous-marine - Territoire.

\section{ABSTRACT}

The organization of scuba diving sites in France: a public and sectoral environmental management of the maritime public domain?

Installing mooring buoys reduces considerably the impact of scuba diving activities on sea-beds. This policy interests scuba divers, managers of scuba diving centers, managers of protected coastal areas, as well as more recently, local councils and associations. However, organizing scuba diving sites could raise sectoral and categorial questions in relation to other activities such as leisure boating and fishing. This raises more general questions about the future of the maritime public domain and its management: should the public sector continue to be responsible for coastal and maritime zones or should privatisation models be adopted?

KEY WORDS : ICZM - Marine Protected Areas - Public Good - Scuba Diving - Territory. 
La fréquentation touristique et récréative du littoral pose de nombreuses questions quant aux problèmes d'impacts sur l'environnement et sur les territoires et quant aux modalités de gestion de l'emprise touristique et de ses effets sur les espaces naturels (Knafou, 1992 ; Cazes, 1992; Deprest, 1997 ; Pinot, 1998; Baron-Yelles, 2001 ; Brigand et Peuziat, 2005). A ce sujet, la plongée sousmarine de loisir, considérée comme une activité de découverte du monde sous-marin, peut avoir des conséquences directes, par «effet-boomerang » (Ribera-Siguan, 1992) sur la faune et la flore marines (Van Treeck et Schuhmacher, 1999; Bellan et Bellan-Santini, 2001). En effet, si la plongée en scaphandre autonome est une activité d'observation à caractère naturaliste, sa pratique à bord de bateaux a pour corollaire, dans la majorité des cas, un ancrage quasi-systématique et répété sur des sites de plongée. Cette question du mouillage, qui concerne également la pratique de la plaisance, est récurrente et s'explique par les impacts produits sur les habitats ou peuplements marins tels que les phanérogames marines (Francour et al., 1999; Milazzo et al., 2004; Peuziat, 2004) ou le coralligène et sur des espèces remarquables à l'instar des gorgones (Ballesteros et al., 1999). Dans d'autres mers, il peut s'agir des récifs coralliens (MPA News, 2005).

La mise en place de bouées d'amarrage réduit considérablement l'impact de la pratique de la plongée sous-marine sur les fonds marins en supprimant les arrachages mécaniques dus au relevage des ancres (Dixon et al., 1993; Francour et al., 2006). Le système le plus couramment utilisé repose sur le principe du corps-mort avec deux différences majeures : l'ensemble est fixé au fond (scellement pour les substrats durs et vis ou enroulement acier pour les substrats meubles et herbiers) et un flotteur intermédiaire de rappel permet d'éviter la destruction de la faune et de la flore liée au poids de la chaîne et de son évitement (fig. 1). Deux autres solutions techniques existent : la bouée d'amarrage peut être située quelques mètres sous la surface (la bouée intermédiaire de rappel n'a plus lieu d'être), ou une simple prise de type organeau sur platine peut être directement scellée à la roche. Dans ces deux cas, l'immersion d'un plongeur est obligatoire pour assurer l'amarrage du bateau.

En France, cette démarche d'équipement est dépendante de plusieurs obligations. Elle nécessite un porteur de projet avec ou sans partenaires. Des budgets sont nécessaires pour acquérir le système adopté, financer sa pose et son entretien, instruire une demande d'Autorisation d'Occupation Temporaire du Domaine Public Maritime (DPM) pour la fixation de la prise au sol et, enfin, s'acquitter de la redevance annuelle associée. L'avis de la Préfecture Maritime relatif à la modification des conditions de navigation et d'ancrage doit également être sollicité. En ce qui concerne la gestion des pratiques subaquatiques, le projet doit reposer sur une démarche de concertation avec les opérateurs de plongée pour identifier les sites à équiper, le nombre de prises d'amarrage par site, les modalités de fonctionnement et les conditions d'accès. En parallèle, l'affectation d'une partie du DPM à une catégorie d'usager ne pouvant être décidée de façon arbitrale, cette consultation doit également associer tous les acteurs de la mer lesquels sont invités à donner formellement leur avis quant à ce partage de l'espace marin avec une validation en Commission Nautique Locale.

Cette démarche d'aménagement des sites de plongée, qui connaît un intérêt croissant sur le littoral français et dans le monde (MPA News, 2005), peut paraître le fruit d'une problématique sectorielle et catégorielle. Si les plongeurs s'impliquent dans ces projets, la raison est simple : la protection des paysages sous-marins, de la faune et de la flore associées, dont il sont les seuls observateurs directs au regard des autres usagers de la mer, permet de garantir une plongée de qualité, un environnement sous-marin préservé et de favoriser un développement durable de leur activité. Au demeurant, l'avènement de ces microterritorialités conduit, en réalité, à s'interroger plus globalement sur les modalités de mise en œuvre et de gestion de ces équipements ainsi que sur le partage de l'espace. Cette politique d'aménagement et de préservation de l'environnement sous-marin peut être interprétée à partir de deux principes de gestion antagonistes : en effet, dans quelle mesure cette démarche de gestion suggère-t-elle une logique de publicisation des territoires marins et sous-marins ou, au contraire, une dynamique de privatisation de biens environnementaux communs par une affectation catégorielle exclusive d'une partie du DPM? 


\section{La complexité réglementaire de l'espace sous-marin}

Dans un premier temps, il est fondamental de mettre en perspective, sur le plan réglementaire et juridique, la question du littoral et de son aménagement, le Droit français ayant permis de consacrer la notion de Domaine Public appartenant aux res communes. Le domaine public rassemble ainsi des biens hors commerce, ne remplissant aucune fonction patrimoniale dans le sens économique, dédiés à l'usage de tous et bénéficiant, de ce fait, d'une protection particulière. L'autorité générale en mer est exercée par l'État, au travers des compétences du Préfet Maritime qui en assure la coordination, en particulier vis-à-vis des Préfets «terrestres », le Préfet de Région étant compétent en matière de pêches maritimes et le Préfet de Département sur le sol et sous-sol. Toutefois, et cette considération est importante au regard des activités balnéaires et des pratiques subaquatiques, l'eau de mer est chose commune, res nullius, non appropriable et ne fait donc pas partie du DPM.

Si le domaine public est par principe imprescriptible et inaliénable, toute occupation doit être obligatoirement précaire et révocable. Sous réserve d'une autorisation soumise à redevance, il est possible d'envisager des aménagements démontables. Cette Autorisation d'Occupation Temporaire $(\mathrm{AOT})$ est délivrée, après visée du dossier, par l'autorité administrative compétente. Cette prise en compte de l'environnement littoral en tant que bien commun relève d'une préoccupation réglementaire marquée qui se prolonge au-delà de l'estran. Ainsi, la loi n 86-2 du 3 janvier 1986 relative à l'aménagement, à la protection et à la mise en valeur du littoral réaffirmet-elle de manière solennelle le principe de libre accès au DPM et responsabilise également le maire d'une commune littorale sur la bande des 300 mètres côté mer. Le Plan Local d'Urbanisme, document d'urbanisme terrestre, doit, depuis les lois de décentralisation et les différentes jurisprudences issues de l'aménagement du littoral et de la « loi littoral », intégrer une partie du DPM immergé. Enfin, avec la loi de démocratie de proximité du 27 février 2002, le champ de compétences du Conservatoire de l'Espace Littoral et des Rivages Lacustres créé en 1975 peut dorénavant être élargi au DPM (espace marin compris) au droit de ses propriétés (Dauvin et al., 2004).

Au regard de l'occupation croissante des littoraux français (Bavoux, 1997; Miossec, 1998a), la France a mené une politique publique de protection du littoral et de l'environnement globalement régalienne et de type «top down » (Prieur, 1980; Beurier et Morvan, 1980; Catanzano et Thebaud, 1995; Féral et al., 1996; Mesnard et Lozachmeur, 2002). Le projet de loi initial relatif à la loi sur les Parcs Nationaux, les Parcs Naturels Régionaux et sur la création d'un nouvel instrument qu'est 
le Parc Naturel Marin, aurait pu modifier en profondeur cette politique publique de conservation définie par un Etat central et reconnue par l'Union Mondiale pour la Nature (UICN). In fine, la loi votée le 14 avril 2006 consacre une nouvelle gouvernance et un engagement public partagé (Mabile, 2006; Romi, 2007). De fait, si les politiques environnementales peuvent être déterminées à partir de concepts scientifiques forgeant des axes prioritaires de recherche, de suivis et de conservation, les modalités effectives de gestion de l'environnement sont également dépendantes de critères idéologiques que la sphère politique et économique définit. Ainsi, la décentralisation et le désengagement progressif de l'État responsabilisent les collectivités territoriales dans la mise en œuvre de politiques publiques environnementales répondant à des exigences nationales et européennes et intégrant de façon croissante la dimension marine des espaces côtiers.

En effet, dans l'ensemble, ces cadres réglementaires relatifs au littoral n'avaient été envisagés qu'à partir de la notion d'un substratum horizontal doublement représenté par la surface de la mer et le plancher marin. Depuis une décennie, la limite terre-mer n'étant plus qu'un artefact obsolète, ce continuum intègre progressivement la dimension sous-marine du littoral, c'est à dire, la colonne d'eau en tant que volume et la vie marine associée. Le littoral devient progressivement un espace qui n'a plus de limite marquée, sa dimension sous-marine étant de plus en plus observée, appréhendée, étudiée (inventaires $\mathrm{ZNIEFF}^{1}$ ) et soumise à des projets territoriaux de gestion et de suivis $\left(\right.$ Liteau $^{2}$, Pnec $\left.^{3}\right)$. Cette évolution est d'ailleurs renforcée par une dynamique européenne très importante de préservation de l'environnement (Directive «Habitats » (1992), le réseau Natura 2000 ou encore les programmes Life ${ }^{4}$ et Inter-Reg ${ }^{5}$ ) et repose sur une vision globale promue lors de la Conférence de Rio de Janeiro en 1992 : la Gestion Intégrée des Zones Côtières (GIZC). Elle structure progressivement toute forme de gestion durable d'un espace littoral (Denis et Henocque, 2001 ; Datar, 2004 ; Pennanguer, 2005). L'appel à projet de la Datar de 2005 contribue à traduire cet impératif de gestion dynamique des littoraux, notamment au niveau des collectivités locales, en encourageant des démarches intégrées et globales prenant en compte la diversité des enjeux et des problématiques relatifs à des territoires littoraux terrestres et marins.

Cette longue mise en perspective réglementaire est fondamentale pour appréhender la dynamique de gestion de l'espace littoral et marin, lequel devient l'objet d'une véritable appropriation scientifique, politique, économique, sociale et par essence, territoriale, au sens de M. Le Berre (1992). Ce mouvement de patrimonialisation émergeant traduit également la mutation d'un espace littoral et marin de moins en moins voué aux pratiques halieutiques professionnelles. Sa conservation ou sa préservation traduit des préoccupations à caractère identitaire, environnemental, social, économique, politique et réglementaire, et cela, de la plus grande échelle à la plus petite. La prise en compte de la globalité des acteurs (Dubrulle et Ghézali, 2002) et des problématiques, en particulier à travers les méthodes de concertation et d'analyse systémique, favorise depuis une décennie cet intérêt pour l'espace marin et sous-marin côtier (Pennanguer, 2005) mais elle illustre également le rôle fondamental des Aires Marines Protégées (AMP) dans ce processus.

\section{Le rôle pionnier des Aires Marines Protégées}

Les AMP sont des espaces fortement prisés par les plongeurs et la problématique de fréquentation soulevée par cette activité récréative était déjà évoquée dans les revues spécialisées dès la fin des années 1970 (Musard, 2003). Depuis le début des années 1990, les pratiques récréatives sont de plus en plus au cœur de la problématique du développement durable de territoires qui ne peuvent être considérés comme étant uniquement des «aires de jeux ». Aussi, pour les ges-

\footnotetext{
1. ZNIEFF : Zones Naturelles d'Intérêt Ecologique, Faunistique et Floristique

2. LITEAU : Programme de recherche du ministère chargé de l'Environnement portant sur les dynamiques et la gestion de l'espace littoral.

3. PNEC : Programme National Environnement Côtier.

4. LIFE : L'Instrument Financier pour l'Environnement.

5. INTER-REG : Programme d'initiative communautaire favorisant la coopération trans-européenne.
} 
tionnaires, il est devenu impératif de se doter de moyens pour quantifier, qualifier et prévenir les différents impacts que peuvent engendrer certaines activités humaines, notamment celles à caractère récréatif. Les études sur la plongée visaient donc surtout à mesurer l'impact écologique des plongeurs et de leur pratique, que ce soit en France (Ballesteros et al., 1999) ou à l'étranger (Davis et Tisdell, 1996; Medio et al., 1997; Rouphael et Inglis, 1997; Hawkins et al., 1999; Jameson et al., 1999; Barker et Roberts, 2004). Parallèlement, les autres travaux effectués sur ce sujet ont consisté à envisager l'impact économique et social de cette activité (Olivier et al., 1992 ; Richez, 1991, 1993; IRAP, 1999), d'autant qu'elle connaissait un essor important à partir de la fin des années 1980 (Livet, 1997).

Ces études et ces suivis ont permis de mettre en œuvre des mesures de gestion appropriées (Francour et al., 2001), à l'instar des bouées d'amarrage, et de développer, au sein même de ces territoires protégés, une prise de conscience accrue quant à la responsabilité de tout un chacun de préserver les sites de plongée (Musard, 2001). Aussi, l'aménagement de sites de plongée concernet-il actuellement et, de manière effective, huit aires marines sur le territoire métropolitain et au moins quatre aires marines au niveau des DOM-TOM. Par ailleurs, deux projets en cours peuvent être évoqués (tableau $1^{6}$ ).

\begin{tabular}{|l|c|c|c|c|}
\hline & $\begin{array}{c}\text { Date de mise } \\
\text { en place }\end{array}$ & $\begin{array}{c}\text { Nombre de } \\
\text { sites }\end{array}$ & $\begin{array}{c}\text { Prises } \\
\text { d'amarrage } \\
\text { disponibles }\end{array}$ & Type d'AMP \\
\hline Direction de l'Agriculture et de la Forêt -Mayotte & 1991 & 7 & 33 & RN \\
\hline Parc National de la Guadeloupe & 1995 & 8 & 27 & PN + RN \\
\hline Parc National de Port-Cros - Port-Cros & 1998 & 8 & 14 & PN \\
\hline $\begin{array}{l}\text { Réserve Naturelle de Saint-Barthélemy } \\
\text { Association GRENAT) }\end{array}$ & 1999 & 20 & 20 & RN \\
\hline Association Parc Marin de la Réunion & 1999 & 41 & 57 & en cours : RN \\
\hline Réserve Naturelle Marine de Cerbère-Banyuls & 2004 & 3 & 15 & RN \\
\hline $\begin{array}{l}\text { Observatoire Marin du SIVOM du Littoral } \\
\text { des Maures }\end{array}$ & 2004 & 1 & 3 & - \\
\hline Réserve Naturelle des Bouches de Bonifacio & 2005 & 1 & $8 * 2$ & RN \\
\hline $\begin{array}{l}\text { Syndicat Intercommunal d'Aménagement } \\
\text { du Golfe du Morbihan }\end{array}$ & 2005 & 1 & 4 & en projet : \\
\hline Conseil Général des Bouches-du-Rhône & 2005 & 8 & $12^{* * *}$ & - \\
\hline $\begin{array}{l}\text { Association de Défense de l'Environnement } \\
\text { et de la Nature des pays d'Agde }\end{array}$ & 2005 & 1 & 8 & $\begin{array}{c}\text { Site Natura } \\
2000\end{array}$ \\
\hline Comm. d'Agglo. Toulon-Provence-Méditerranée & 2006 & 16 & 16 & - \\
\hline Association Plongeurs de l'Est-Var & En projet & 1 & dizaine & - \\
\hline Parc National de Port-Cros - Porquerolles & En projet & 11 & 11 & $\begin{array}{c}\text { Site Natura } \\
2000\end{array}$ \\
\hline
\end{tabular}

PN : Parc National; RN : Réserve Naturelle; PNR : Parc Naturel Régional

* Bouées d'amarrage situées à quelques mètres sous la surface

** Prises d'amarrage fixées sur le fond

Tableau 1 : Les aires marines concernées par l'aménagement des sites de plongée depuis 15 ans en France (DOMTOM compris)

The Marine Areas concerned by mooring buoys for scuba diving sites for the last 15 years in France (DOMTOM included)

6. Le tableau 1 est issu d'une enquête nationale envoyée en février 2006 pour le Conservatoire-Études des Ecosystèmes de Provence et dont les résultats intéressent également le Forum des Aires Marines Protégées [http://www.airesmarines.org] et présentés sur le site du réseau MEDPAN [http://www.medpan.org]. Que toutes les personnes et structures ayant renseigné cette enquête soient ici remerciées. Aucune donnée n’a pu être recueillie pour la Polynésie française et la Nouvelle-Calédonie. 
Si ce mouvement s'accélère nettement depuis 2004, il est initialement le fait des gestionnaires de territoires protégés reconnus comme un patrimoine commun et comme un bien public au sens de res publicae. Globalement, cette démarche pionnière est facilitée par la réunion de quatre facteurs inhérents au fonctionnement d'une AMP, laquelle est définie par un territoire délimité, un statut réglementaire associé, un gestionnaire identifié et identifiable et la possibilité de recourir à des instruments financiers et techniques adaptés pour répondre aux engagements fixés par un plan de gestion validé.

Les gestionnaires de ces AMP ont su s'adapter à l'évolution de la société post-industrielle et ont développé, les premiers, des outils appropriés de gestion et d'ouverture au public. Si les AMP apparaissent comme les vitrines de ce que la nature a été ou de ce qu'il faudrait qu'elle soit partout ailleurs et comme un patrimoine national pour lequel la force de l'action publique a préempté de façon réglementaire sur les logiques privées, elles renvoient, pour une large part, à la manière dont se dessinent les projets de société (WCPA, 1997). Contrairement aux analyses de A. Vallega (1996) reprises par A. Miossec (1998a ; 1998b) considérant les AMP comme une contrainte quasi-systématique pour les activités marines et littorales et comme une source de conflits potentiels stimulant les antagonismes entre usagers, les AMP sont surtout « des lieux exemplaires, construits pour signifier la possibilité d'un avenir différent, articulés à la désignation concomitante d'un problème social, d'une contradiction [...] et enfin, construits pour être reproduits et imités » (Micoud, 1991). Avec des finalités partiellement différentes, cette démarche de gestion catégorielle, restreinte à des micro-territoires protégés, peut dorénavant être reproduite par des collectivités compétentes dans le cadre des outils territoriaux actuels de gestion intégrée.

\section{Légitimité économique et projet de territoire : les enjeux d'une gestion}

Au sein des AMP, l'intégration de la variable économique, comme critère d'évaluation des effets de la protection de la nature, a permis de mieux légitimer l'intérêt de ces politiques de gestion des territoires et de valoriser les biens communs de l'environnement (Miossec, 1998b). Cette reconnaissance économique a ainsi permis de forger l'idée selon laquelle ces biens patrimoniaux (Clayes-Mekdade et al., 1999), également définis comme des actifs environnementaux ou un « capital-nature », étaient dotés d'une valeur d'existence ou de legs (Boisvert et Vivien, 1998), au sein des AMP ou en dehors. Ce processus et cette typologie des valeurs, basés sur des schémas socio-culturels (De Montgolfier et Natali, 1987), conduisent progressivement à définir une représentation factuelle et structurée de la dimension sous-marine du littoral en tant que « chose publique ». La mécanique d'un «jeu social » s'est donc amorcée en dehors des AMP et permet une confrontation entre acteurs, suivant des logiques raisonnées, conscientes et inconscientes (Mermet, 1992 ; Rousseau et Deffuant, 2005) et des légitimités différentes (Godard, 1989; Catanzano et Thebaud, 1995).

La démarche d'équipement des sites de plongée, particulièrement importante pour les années 2005 et 2006, souligne ainsi l'intérêt partagé des différents acteurs locaux pour mettre en ouvre ces solutions a priori simples de préservation de l'environnement marin et de gestion des usages. En dehors du SIVOM du littoral des Maures ayant équipé une épave, ces dispositifs d'amarrage sont installés pour protéger des habitats et des espèces remarquables répondant ainsi à des stratégies de mise en valeur, de gestion et de conservation différentes : l'encadrement de l'activité de plongée sous-marine par la mise en place de bouées d'amarrage peut autoriser une augmentation significative de la fréquentation ou une régulation par le biais de l'établissement d'un numerus clausus; le sacrifice d'un ou plusieurs sites peut également constituer une solution adaptée pour les gestionnaires, à condition que ces options soient envisagées dans le cadre de l'élaboration d'un contexte réglementaire approprié et par la diffusion d'une information pédagogique et de sensibilisation (Dixon et al., 1993; Davis et Tisdell, 1996; Musard, 2003).

Par conséquent, ces équipements s’intègrent à des dispositifs réglementaires, financiers ou de gestion territoriale particuliers. Chaque organisme institutionnel répond à sa vocation première : la protection de la nature ou l'élaboration d'un projet de territoire à partir d'un instrument juridi- 
que national ou européen. Par exemple, les aménagements réalisés à la Réunion ou dans le Golfe du Morbihan participent d'un projet de territoire affirmé puisque l'Association Parc Marin de la Réunion et le Syndicat Intercommunal d'Aménagement du Golfe du Morbihan s'engagent dans un processus de mise en protection d'aires marines, avec respectivement, la création d'une Réserve Naturelle marine (officiellement créée par le décret 2007-236 du 21 février 2007) et la création d'un Parc Naturel Régional. En Guadeloupe et à Mayotte, la logique est similaire.

Cette dynamique d'aménagement nécessite une prise de décision politique de la part des gestionnaires ou des élus pour contribuer à la réalisation de ces projets par des investissements en majorité publics et para-publics (du niveau local au niveau européen). Il s'agit d'une démarche de gestion publique d'un bien commun inaliénable et, qui plus est, sans aucune compensation financière directe attendue. La gratuité d'accès est, pour l'instant, garantie partout à l'exception de la Réserve Naturelle de Saint-Barthélemy : les gestionnaires y sollicitent les structures commerciales de plongée à hauteur de 1 euro par plongeur et par jour pour auto-financer ces équipements. À terme, cette question d'une éco-taxe ou d'une participation pourrait néanmoins être posée sur la base du principe du consentement à payer (Arin et Kramer, 2002) d'autant que le principe de ces aménagements bénéficie en grande partie à la seule activité économique de plongée sous-marine.

\section{L'appropriation privative ou les limites d'une solution idéologique}

Ainsi, dans le cas de Saint-Raphaël, l'aménagement du site de plongée « le Lion de Mer », réalisé sans aucune AOT, a été décidé par les opérateurs de plongée depuis une dizaine d'années, avec néanmoins, un accord de principe de la part des pêcheurs professionnels. Chaque structure de plongée possède une bouée dont la fixation sur le fond est assurée par des chaînes entourant des rochers ou par des corps-morts. L'objectif commun à l'association des Plongeurs de l'Est-Var et la commune de Saint-Raphaël est de proposer des aménagements en conformité avec les contraintes réglementaires et environnementales. Pour autant, des questions relatives à l'affectation de la gestion des équipements et à la possible mise en œuvre d'une amodiation ralentissent l'instruction du dossier et le projet. Cet exemple illustre l'intérêt de ces opérateurs de plongée et de cette collectivité pour promouvoir une gestion légalisée des sites de plongée. Néanmoins, cette démarche n'est pas répandue car elle implique lourdeurs administratives et incertitudes financières : nombre de sites de plongée restent ainsi illégalement aménagés par des opérateurs de plongée attentifs à leur rentabilité économique et à la préservation de l'environnement.

Ces constats évoquent de façon singulière le problème de l'usage des biens communs telles que les ressources naturelles, théorisé par Garrett Hardin en 1968 sous le nom de «tragedy of the commons » ( tragédie des communs » ou " tragédie des communaux »). Véritable mythe fondateur de l'«écologie de marché »(Ost, 1995), cette théorie a permis de développer une analyse néolibérale en faveur de la privatisation de l'environnement comme seul moyen efficient pour éviter la dilapidation de ressources naturelles en accès libre et ayant un marché. Malgré des présupposés empiriquement faux et des contre-vérités historiques, cette importante théorie sur l'appropriation, l'usage individuel et collectif et la nécessaire privatisation d'un bien commun continue à marquer les esprits (Aubertin et al., 1998; Boisvert, 2003; Boisvert et al., 2004). Au-delà de son caractère idéologique, elle permet de caractériser et de développer de façon plus fine une réflexion sur les «modes d'appropriation » (Weber et Réveret, 1994) des territoires et sur leur gestion intégrée ou catégorielle.

L'organisation des mouillages et le processus d'appropriation territoriale déterminé par l'implantation et l'organisation de pratiques subaquatiques soumises à concurrence sur un bien commun (un site de plongée reconnu pour sa valeur d'usage, biocénotique ou patrimoniale), pourraient ainsi être appréciés à partir du modèle théorique de Hardin. Néanmoins, une telle analyse légitimant in fine la privatisation des sites aménagés souffrirait d'indigence si elle n'était pas pondérée par deux constats, lesquels renvoient pour partie à certaines des critiques formulées à l'encontre des postulats initiaux de Hardin. 
Tout d'abord, les données scientifiquement valides hors AMP démontrant la dégradation des sites de pratiques subaquatiques en Méditerranée française due à une pression croissante de la part des opérateurs de plongée manquent (Zabala, 1999). De fait, la privatisation des sites de plongée, comme solution de gestion hors AMP, ne se justifie pas au regard du manque de recul scientifique concernant les processus de dégradation et de résilience des paysages sous-marins.

En second lieu, contrairement à la théorie de Hardin où un seul groupe économique exploite de façon exclusive une ressource singulière sur un territoire donné, ces aires marines sont fréquentées par d'autres groupes d'usagers suivant des logiques d'usages différentes. Ce type d'aménagement et de gestion du DPM requiert, assurément, l'avis de tous les acteurs de la mer, rendant le processus de concertation et d'installation plus difficiles. Par exemple, les pêcheurs professionnels sont, en général, assez réticents au principe des bouées d'amarrage : elles marquent et définissent le territoire des plongeurs et peuvent les en exclure. La gêne occasionnée par ces équipements se transforme potentiellement en un sentiment de spoliation, cristallisant l'achèvement d'une mutation socio-spatiale du littoral.

\section{La contractualisation ou le principe de «bonne gouvernance "}

Aussi, pour mener à bien ces projets d'aménagement avec pour corollaire une autorégulation acceptée et un accord de la part des autres usagers, le recours à un conciliateur est nécessaire pour motiver et susciter les convergences d'intérêts entre écologie, économie et principe d'accès au DPM. En effet, concernant les douze aires marines où sont installées des bouées destinées aux plongeurs, pour neuf organismes, cette politique d'aménagement répond à une volonté conservatoire de gestion, pour sept d'entre eux, à une demande des plongeurs et pour trois d'entre eux, à une étude de fréquentation. En fait, dans un système catégoriel d'acteurs articulé sur la seule activité de plongée, chacun des agents socio-économiques revendique et entretient une relation plus ou moins étroite aux territoires sous-marins (fig. 2).

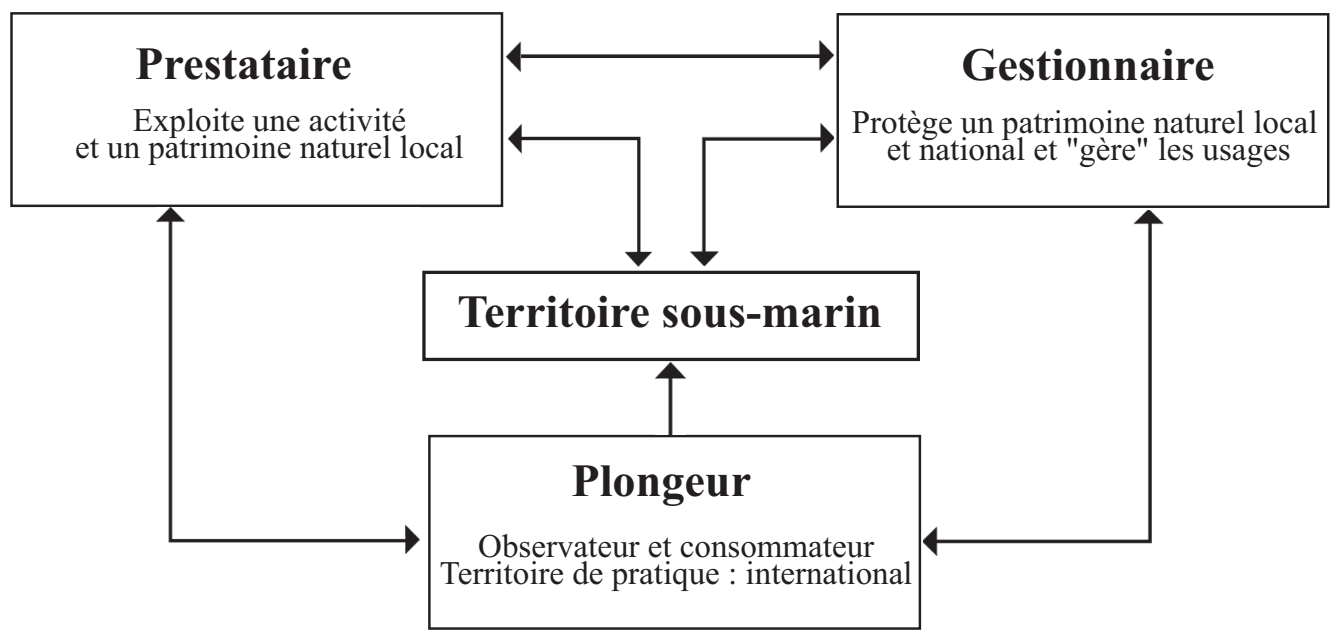

Figure 2 : Une relation interdépendante au territoire sous-marin An interdependant relationship to the submarine territory 
Il apparaît, dans le cas d'un territoire sous-marin à caractère touristique, que les plongeurs peuvent connaître une faible dépendance au patrimoine naturel local puisqu'ils peuvent être de passage et que leur territoire de pratique est étendu. Néanmoins, cet environnement sous-marin peut s'inscrire dans la logique des prestataires de plongée qui, s'ils ne considèrent pas ces territoires de pratiques comme uniquement un outil de travail et un capital naturel à gérer, peuvent également le considérer comme un patrimoine naturel, facteur d'identité territoriale (Musard, sous presse). De fait, ensemble, ils peuvent participer et contribuer à la préservation de ces territoires sous-marins et adhérer, le cas échéant, à la démarche des gestionnaires d'aires marines. Ces derniers, en proposant des politiques scientifiques et des stratégies d'encadrement de la fréquentation adaptées, tentent de le préserver en intégrant les différentes logiques d'usages afférentes à ce territoire sous-marin dont ils ont la charge réglementairement. Pour autant, l'installation de bouées d'amarrage ne doit pas occulter l'impact potentiel des plongeurs, lesquels doivent aussi modifier leurs pratiques et leurs comportements (Javel et al., 2006) pour que cette politique conservatoire soit efficace. Dans ce système d'acteurs, les responsabilités se partagent et s'assument pour une prise en compte pérenne de l'évolution des territoires et du patrimoine naturel local. Ce bien commun est géré de façon commune à partir d'une plate-forme de travail et de concertation qui prend la forme d'un acte d'engagement à caractère contractuel.

En effet, les modalités de gouvernance et de mise en projets de territoires se basent depuis quelques années sur des principes de concertation. La législation française étant peu contraignante sur un certain nombre d'aspects relatifs à l'encadrement de la fréquentation touristique dans les espaces naturels protégés (Gérardin, 1999), définir un contrat de confiance ou de partenariat s'est imposé au regard du «[...] souci partagé d'assurer la pérennité d'un milieu naturel, culturel et paysager d'exception et d'une activité de plongée de découverte, de reconnaissance et de contribution à la gestion patrimoniale des fonds marins » comme le souligne le préambule de la charte de plongée de Port-Cros de 2006. Le développement de ces chartes ( 5 en 2003, 9 en 2006) favorise l'idée selon laquelle la négociation est largement au cœur des modalités de gouvernance et de gestion pour favoriser collectivement de nouvelles coopérations et l'adoption de comportements plus respectueux.

Chronologiquement, les chartes de plongée mises en œuvre au sein des AMP de Méditerranée française l'ont été avant l'installation des bouées d'amarrage. Sur ces territoires, les gestionnaires et les opérateurs ont pu établir un dialogue constructif autour d'un projet commun. Hors AMP, la négociation est plus difficile car il n'y a ni territoire borné, ni statut réel en dehors de la notion de DPM ou des attributions de compétences particulières, ni de véritable gestionnaire et les responsables de centres de plongée sont dans une dynamique d'exploitation commerciale du patrimoine naturel. Suggérer un engagement, solliciter une démarche éthique et responsable de la part des plongeurs se révèle aléatoire s'il s'agit d'un engagement unilatéral sans aucune contre-partie. Pour ces raisons, hors AMP, la mise en place de bouées d'amarrage est une condition sine qua non à la formalisation et l'adoption de chartes de plongée.

Pour autant, si cette différence entre aires marines protégées et non protégées est importante sur le plan de la cinétique de la gouvernance, elle n'affecte pas la valeur de l'engagement commun et la vision d'avenir que la charte structure et développe. En effet, dans l'ensemble, la négociation a permis de promouvoir un cadre contractuel basé sur le respect des engagements, mutuellement consentis, signés et adoptés, afin que se rejoignent durablement les intérêts des gestionnaires (la protection de la nature, l'accueil du public et l'image de l'aire marine), des opérateurs de plongée (un « capital nature » préservé) et des plongeurs (concordance des attentes et des représentations). La charte repose sur un contrat de confiance et défend une logique partenariale autour d'un projet commun. Formalisée, adoptée et appliquée, elle n'est pas pour autant figée. La discussion collective et l'empirisme fixant l'esprit de la charte, elle évolue en fonction des impératifs et des besoins de chacun des partenaires.

Ce mode de gouvernance entre le gestionnaire, l'opérateur de plongée et le plongeur permet une véritable concertation concernant le devenir d'un territoire et d'une pratique et devient un outil de 
régulation intégré au dispositif de gestion et de conservation. Ainsi, la notion de numerus clausus peut-elle figurer dans le texte d'une charte comme celle de Port-Cros : la capacité de charge de quelques sites de plongée est énoncée avec un maximum de 40 plongeurs autorisés à s'immerger simultanément. C'est la force de ces « textes faibles » (Dourlens, 2000) qui se développent à partir de suggestions simples et évoluent vers un cadre de contraintes volontairement accepté et négocié entre les différentes parties pour aboutir à des résultats particulièrement innovants. Ainsi, la première charte de Port-Cros a-t-elle été signée en 1994 par quelques structures de plongée locales qui devenaient «partenaires ». Après différentes périodes de négociation, en 2004, la signature de cette charte est devenue obligatoire pour tout plongeur souhaitant fréquenter les eaux du Parc National. Autre exemple, en 2006, la charte de plongée du conseil général des Bouches-du-Rhône a formalisé une démarche transversale dans laquelle s'investissaient les clubs de plongée locaux et les pêcheurs professionnels ayant favorisé ce projet d'envergure.

Il est enfin important de souligner la démarche défendue par l'association Longitude 181 depuis 2002. Dans la veine de la «Charte Éthique du Voyageur» créée par le tour-opérateur Atalante, la « Charte Internationale du Plongeur Responsable » que cette association promeut, est reconnue par une grande majorité des écoles de plongée à travers le monde et traduite en douze langues. Elle est adoptée par des centre de plongée ambassadeurs et sollicite l'adhésion de plongeurs sur des questions liées à la plongée et à la préservation de l'environnement sous-marin en suscitant, par exemple, l'installation de bouées d'amarrage. Elle suggère, plus globalement, une réflexion au-delà de la seule activité de plongée. La charte véhicule des notions d'engagement vis-à-vis des populations locales, ou encore, pose le problème de la consommation partagée des ressources naturelles : l'eau douce, par exemple, bien pouvant être rare, peut être utilisée de façon disproportionnée par les plongeurs, notamment pour le rinçage du matériel. Cette association se fixe des objectifs d'éducation, d'adhésion et de respect qui transcendent la singularité d'un territoire sous-marin. Les notions de développement durable et de tourisme durable, de gestion des territoires et de préservation du patrimoine naturel tendent ainsi à se diffuser de plus en plus parmi les adeptes de la plongée sous-marine.

\section{Conclusion}

L'aménagement des sites de plongée pose la question de l'appropriation d'une portion de territoire marin et sous-marin au sens qu'il s'agit d'une entité littorale et marine, circonscrite et choisie pour sa fonctionnalité dont la dimension sous-marine est reconnue pour ses qualités biocénotiques, paysagères et patrimoniales. Elle fait donc l'objet d'une anthropisation temporaire basée sur des valeurs socio-économiques, identitaires, éducatives ou symboliques et consacrée par des aménagements, des types de pratiques et des modes de gestion adaptés (Musard, 2003). Cette appropriation relève ainsi d'une démarche de gestion pour laquelle un agent qualifié de « gestionnaire » développe un projet de territoire auquel participent tous les usagers de la mer et acteurs institutionnels à travers un processus de concertation obligatoire. Si ce gestionnaire est responsable d'une aire marine sous statut de protection nationale, il est dès lors reconnu comme l'interlocuteur incontournable. Si ce gestionnaire s'investit sur une aire marine non protégée dont le devenir est inscrit dans des projets de territoires transversaux et intégrés, il doit promouvoir et faire reconnaître institutionnellement sa démarche de préservation et de gestion, assumer le rôle de leader, de médiateur et d'intermédiaire et engager des actions concrètes.

La demande croissante en équipements de sites de plongée et ce rôle de substitution assumée par des structures gestionnaires de type Établissement Public, Collectivité Territoriale ou Association de Protection de la Nature pour conduire ces projets territoriaux à leurs termes, confirment que cette question relève d'une politique de publicisation des espaces naturels basée sur l'intérêt général quant à la conservation d'un bien commun. Cette affectation d'une partie du DPM, même catégorielle et quasi-exclusive, responsabilise et mandate les prestataires de plongée au regard des autres acteurs de la mer et de cette exigence sociétale partagée quant au devenir de ces micro-territoires. Actuellement, en dehors de cas d'aménagements illégaux stricto sensu, la 
logique de privatisation ne peut donc se développer véritablement sur une zone côtière dont les cadres réglementaires affirment l'importance du bien commun ou de la chose publique qu'il est nécessaire de préserver par l'intermédiaire d'un gestionnaire qualifié. Ce type de démarche s’inscrit dans un mouvement de valorisation des actifs environnementaux et de l'efficience sociale, environnementale, économique et politique de l'action publique (Maresca et al., 2006). Néanmoins, à terme, hors AMP, la gestion des bouées d'amarrage pourrait être déléguée : l'impulsion d'une telle démarche relève d'une exigence collective envers la préservation d'un patrimoine commun mais pour assurer la pérennisation de ce type d'équipement, une délégation de service public pourrait éventuellement être mise en œuvre.

Ces réflexions permettent également de considérer la question de l'aménagement des sites plongée comme relevant d'une démarche constitutive de la Gestion Intégrée des Zones Côtières. Cette démarche conduit en effet à structurer et défendre un mode de gouvernance territoriale pour laquelle les fondamentaux du concept de développement durable s'imposent. Ces derniers incitent tous les acteurs présents sur un territoire à se concerter et à s'organiser afin de conduire des projets dans le cadre des réalités socio-spatiales et dans le respect des aspects environnementaux et réglementaires. En s'intéressant au problème des bouées d'amarrage pour la plongée sous-marine, diagnostics, inventaires et processus de concertation favorisent l'émergence d'une lecture transversale des problématiques, des acteurs et des enjeux économiques et environnementaux au niveau des différents cadres réglementaires nationaux et européens. La dimension a priori catégorielle de cette question s'inscrit donc dans une démarche de gestion globale et favorise techniquement la réalisation d'un segment de toute politique de gestion intégrée de la zone côtière tout en stimulant les synergies et les acteurs vers un développement durable du littoral, y compris dans sa dimension sous-marine.

\section{Bibliographie}

Arin (T.), Kramer (A. R.), 2002. - « Divers' willingness to pay to visit marine sanctuaries: an exploratory study », Ocean E Coastal Management, 45, p. 171-183.

Aubertin (C.), Boisvert (V.), Vivien (F.-D.), 1998. - « La construction sociale de la biodiversité », Natures Sciences Sociétés, Vol. 6, n 1, p. 7-19.

Ballesteros (E.), Coma (R.), Dantart (L.), Dies (D.), Behereu (B.), Martiny de Maissonave (L.), Zabala (M.), 1999. - Étude de l'impact de la plongée sous-marine sur le coralligène. Protocole d'observation et études comparatives entre espaces protégés, Université de Barcelone/Parc National de Port-Cros, Contrat 98029, Barcelone, 27 p.

BARKer (N. H. L.), RoberTS (C. M.), 2004. - « Scuba diver behaviour and the management of diving impacts on coral reefs », Biological Conservation, 120, p. 481-489.

BARON-Yelles (N.), 2001. - «Tourisme et aires protégées du littoral : le cas de la façade atlantique française », L'Information Géographique, vol. 65, p. 141-155.

Bavoux (J.-J.), 1997. - Les littoraux français, Paris, A. Colin, coll. «U Géographie », 268 p.

Bellan (G.), Bellan-Santini (D.), 2001. - «A review of littoral tourism, sport and leisure activities : consequences on marine flora and fauna », Aquatic Conserv. Mar. Freshw. Ecosyst., 1 1, p. 325-333.

Beurier (J.-P.), Le Morvan (D.), 1980. - « Quelques réflexions sur le concept de parc marin en droit français », Revue Juridique de l'Environnement, $\mathrm{n}^{\circ}$ 4, p. 318-336.

Boisvert (V.), Caron (A.), Rodary (E.), 2004. - « Privatiser pour conserver? Petits arrangements de la nouvelle économie des ressources avec la réalité », Revue Tiers-Monde, 177, p. 60-83.

Boisvert (V.), 2003. - « Le débat sur les droits de propriété dans les négociations sur la biodiversité », dans Serfati (C.) (dir.), Enjeux de la mondialisation : un regard critique, Toulouse, Octares, coll. "Colloque », p. 117-128. 
Boisvert (V.), Vivien (F.-D.), 1998. - « Un prix pour la biodiversité. L'évaluation économique entre différentes légitimités », Natures Sciences Sociétés, Vol. 6, n² 2, p. 17-26.

Brigand (L.), Peuziat (I.), 2005 - « Nautisme et fréquentation des espaces sensibles. Le cas des îles et des archipels de la France métropolitaine », dans Bernard (N.) (dir.), Le Nautisme, acteurs, pratiques et territoires, Rennes, PUR, coll. «Espace et territoires », p. 301-319.

Catanzano (J.), Thebaud (O.), 1995. - Le littoral. Pour une approche de la régulation des conflits d'usage, Paris, Institut Océanographique/IFREMER, coll. «Propos », 149 p.

CAZES (G.), 1992. - Fondements pour une géographie du tourisme et des loisirs, Paris, Bréal, coll. « Amphi Géographie », 189 p.

Clayes-Mekdade (C.), Géniaux (G.), Luchini (S.), 1999. - «Approche critique et mise en œuvre de la méthode d'évaluation contingente : un dialogue entre économiste et sociologue », Natures Sciences Sociétés, Vol. 7, n² 2, p. 35-47.

DATAR (2004). - Construire ensemble un développement équilibré du littoral, Paris, La Documentation française, coll. «Études et Recherches », 156 p.

Dauvin (J.-C.), Lozachmeur (O.), Capet (Y.), Dubrulle (J.-B.), Ghezali (M.), Mesnard (A.-H.), 2004. - «Legal tools for preserving France's natural heritage through integrated coastal zone management », Ocean E Coastal Management, 47, p. 463-477.

Davis (D.), Tisdell (C.), 1996. - « Economic Management of Recreational Scuba Diving and the Environment », Journal of Environmental Management, 48, 3, p. 229-248.

De Montgolfier (J.), Natali (J.-M.), 1987. - Le patrimoine du futur. Approches pour une gestion patrimoniale des ressources naturelles, Paris, Economica, coll. «Économie agricole et agroalimentaire », 248 p.

Denis (J.), HÉnocque (Y.), 2001. - Des outils et des hommes pour une gestion intégrée des zones côtières, Guide méthodologique, volume II, UNESCO, Paris, Manuels et Guides (COI ; 42), 62 p.

Deprest (F.), 1997. - Enquête sur le tourisme de masse. L'écologie face au territoire, Paris, Belin, coll. «Mappemonde », 207 p.

Dixon (J. A.), Scura (L. F.), VAn'T Hof (T.), 1993. - « Meeting ecological and economic goals: marine parks in the Caribbean », Ambio, 22, p. 117-125.

Dourlens (C.), 2000. - «Action collective, engagements privés : la régulation par les chartes », dans Micoud (A.), Peroni (M.), Ce qui nous relie, La Tour-d'Aigues, Édition de L'Aube, coll. « Société et territoire », p. 317-332.

Dubrulle (J.-B.), GhÉZAli (M.), 2002. - «Analyse institutionnelle des acteurs impliqués dans la connaissance et la gestion du littoral », dans Dauvin (J.-C.) (dir.), Gestion intégrée des zones côtières : outils et perspectives pour la préservation du patrimoine naturel, Paris, Muséum National d'Histoire Naturelle, coll. «Patrimoines Naturels », p. 109-130.

Féral (F.), Mamontoff (C.), Rouquan (O.), 1996. - Le littoral. L'Etat régulateur : droit domanial et stratégies politiques, Institut Océanographique/IFREMER, Paris, collection « Propos », 125 p.

Francour (P.), Ganteaume (A.), Poulain (M.), 1999. - « Effects of boat anchoring in Posidonia oceanica seagrass beds in the Port-Cros National Park (North-Western Mediterranean Sea)», Aquatic Conserv. Mar. Freshw. Ecosyst., 9, p. 391-400.

Francour (P.), Harmelin (J.-G.), Pollard (D.), Sartoretto (S.), 2001. - «A review of marine protected areas in the Northwestern Mediterranean region: siting, usage, zonation and management », Aquatic Conser. Mar. Freshw. Ecosyst., 11, p. 155-188.

Francour (P.), Magreau (J.-F.), Mannoni (P.-A.), Cottalorda (J.-M.), Gratiot (J.), 2006. - Ancrages écologiques permanents. Guide d'Aide à la Gestion des Aires Marines Protégées, Nice, Université de NiceSophia Antipolis/Parc National de Port-Cros, 68 p.

GÉRARDin (N.), 1999. - "L'île en partage, la gestion des conflits d'usage par le parc national de Port-Cros », Cahier espaces, $n^{\circ}$ 62, p. 29-31.

Godard (O.), 1989. - « Jeux de nature : quand le débat sur l'efficacité des politiques publiques contient la question de leur légitimité », dans Mathieu (N.), Jollivet (M.) (dir.), Du rural à l'environnement, Paris, ARF/L'Harmattan, coll. « Bibliothèque des ruralistes », p. 303-342.

Hardin (G.), 1968. - « The tragedy of the commons », Science, 162, p. 1243-1248. 
Hawkins (J.P.), Roberts (C. M.), Van’t Hof (T.), de Meyer (K.), Tratalos (J.), Aldam (C.), 1999. «Effects of recreational scuba diving on Caribbean coral and fish communities », Conservation Biology, $13, n^{\circ} 4$, p. 888-897.

IRAP, 1999. - Etude des retombées du Parc National sur l'activité économique et sur l'emploi, Rapport final, IRAP/Parc National de Port-Cros, Annecy, 76 p.

Jameson (S.C.), Ammar (M.S.A.), Saadalla (E.), Mostafa (H.M.), Riegl (B)., 1999. - «A coral damage index and its application to diving sites in the Egyptian Red Sea », Coral Reefs, 18, p. 333-339.

Javel (F.), Riocreux (F.), Musard (O.), Pironneau (E.), 2006. - Étude de limpact potentiel de la plongée sousmarine dans la réserve naturelle marine de Cerbère-Banyuls (zone de mouillages organisés du Cap l'Abeille), Aix-en-Provence, Contrat Conseil Général des Pyrénées-Orientales/SAFEGE Environnement, 46 p.

Knafou (R.), 1992. - «L'invention du tourisme », dans Bailly (A.), Ferras (R.), Pumain (D.) (dir.), Encyclopédie de géographie, Paris, Economica, p. 851-864.

Le Berre (M.), 1992. - «Territoire », dans Bailly (A.), Ferras (R.), Pumain (D.) (dir.), Encyclopédie de géographie, Paris, Economica, p. 617-638.

Livet (R.), 1997. - « De la plongée sportive au tourisme sous-marin », Cahier Espaces, n 52, p. 62-68.

Mabile (S.), 2006. - «Les parcs naturels marins consacrés par le législateur », Revue Juridique de l'Environnement, 3, p. 251-264.

Maresca (B.), Ranvier (M.), Dujin (A.) - 2006. Valoriser l'action publique. Le " consentement à payer », un outil au service de la LOLF, CREDOC, Paris, Cahier de Recherche, n²24, 126 p.

Medio (D.), Ormond (R. F. G.), Pearson (M.), 1997. - « Effect of briefings on rates of damage to corals by scuba divers ", Biological Conservation, 79, p. 91-95.

Mermet (L.), 1992. - Stratégies pour la gestion de l'environnement - La Nature comme jeu de société, Paris, L'Harmattan, coll. « Environnement », 205 p.

Mesnard (A.-H.), Lozachmeur (O.), 2002. - « La protection du patrimoine naturel du littoral au niveau national », dans Dauvin (J.-C.) (dir.), Gestion intégrée des zones côtières : outils et perspectives pour la préservation du patrimoine naturel, Paris, Muséum National d'Histoire Naturelle, coll. "Patrimoines Naturels », p. 84-108.

Micoud (A.) (dir.), 1991. - Des hauts lieux. La construction sociale de l'exemplarité, Paris, CNRS, 133 p.

Milazzo (M.), Badalamenti (F.), Cecchereldi (G.), Chemello (R.), 2004 - « Boat anchoring on Posidonia oceanica beds in a marine protected area (Italy, western Mediterranean): effect of anchor types in different anchoring stages », Journal of Experimental Marine Biology and Ecology, 299, p. 51-62.

Miossec (A.), 1998a. - Les littoraux, entre nature et aménagement, Paris, SEDES, coll. « Campus Géographie », $192 \mathrm{p}$.

—, 1998b. - « De l'aménagement des littoraux à la gestion intégrée des zones côtières », dans MiossEC (A.) (dir.), 1998, Géographie humaine des littoraux maritimes, Paris, CNED-SEDES, coll. « CNED-SEDES Concours ", p. 413-466.

MPA News, 2005. - Vol. 7, n 3, [http://depts.washington.edu/mpanews/MPA67.htm].

Musard (O.) (sous presse) - " "Mérouville" ou la marchandisation de l'identité de la Corse sous-marine " dans Baron-Yelles (N.) (dir.). La Méditerranée, espace physique, espace vivant, Actes du $128^{\mathrm{e}}$ Congrès du CTHS «Relations, échanges et coopération en Méditerranée », Bastia, 14-21 avril 2003.

—, 2003. - Les pratiques subaquatiques au sein des aires marines protégées de Méditerranée française : entre paysages sous-marins, représentations et impacts. Contribution au développement d'une géographie relative aux territoires sous-marins, Thèse de doctorat de géographie, Aix-Marseille I - Maison Méditerranéenne des Sciences de l'Homme, Aix-en-Provence, 449 p.

—,2001. - La plongée sous-marine au sein de la Réserve Naturelle des Bouches de Bonifacio : l'offre, la demande, les problématiques, Aix-en-Provence, Contrat Association Pour les Universités Rurales Européennes/Office de l'Environnement de la Corse, $171 \mathrm{p}$.

Olivier (J.), Girardin (N.), Jeudy de Grissac (A.), 1992. - Impact économique des espaces côtiers protégés de la Méditerranée, Colloque organisé à Ajaccio, 26-28 septembre 1991, News MEDPAN, Secrétariat du MEDPAN, Parc National de Port-Cros, n³ 3, juin 1992, Hyères, 98 p. 
Ost (F.), 1995. - La nature hors la loi, l'écologie à l'épreuve du droit, Paris, La Découverte, coll. « Textes à l'appui », 346 p.

Pennanguer (S.), 2005. - Incertitude et concertation dans la gestion de la zone côtière, Thèse de doctorat ENSAR, Rennes, Ecole Nationale Supérieure Agronomique de Rennes/Agrocampus, 372 p.

Peuziat (I.), 2004. - «Plaisanciers en quête d'espaces naturels et de tranquillité : illusion ou réalité? Le cas de l'archipel de Glénan (France) », Norois, n 193-2004/4, p. 103-115.

PINOT (J.-P.), 1998. - La gestion du littoral, t. 1 : Littoraux tempérés, côtes rocheuses et sableuses, Paris, Institut Océanographique, coll. «Propos », 400 p.

Prieur (M.), 1980. - « Le droit des aires marines protégées en France », Revue Juridique de l'environnement, $n^{\circ} 4$, p. 337-342.

Ribera-Siguan (M.-A.), 1992. - La réserve marine des îles Medes. Bilan d'un succès imprévu, dans Parchi marini del Mediterraneo, Atti del $2^{\circ}$ Convegno internazionale, 17-19 May 1991, San Teodoro, I.CI.MAR publ., p. 152-161.

Richez (G.), 1991. - La fréquentation du Parc National de Port-Cros par les plongeurs sous-marins - apnée exclue - durant l'été 1990, Rapport au Parc National de Port-Cros, Hyères, 65 p.

—, 1993. - « La plongée sous-marine de loisir en Corse - apnée exclue - durant l'été 1991 », Trav. sci. Parc nat. rég. Rés. nat. Corse, $\mathrm{n}^{\circ}$ 45, $65 \mathrm{p}$.

Romi (R.), 2007. - «Parcs nationaux : fin ou renouveau d'un modèle juridique? », Revue Juridique de l'Environnement, 1, p. 37-52.

Rouphael (A. B.), Inglis (G. J.), 1997. - « Impacts of recreational scuba diving at sites with different reef topographies », Biological Conservation, 82, p. 329-336.

Rousseau (L.), Deffuant (G.), 2005. - «Gestion des territoires : aider à la formulation collective de problèmes », Nature Sciences Sociétés, 13, p. 21-32.

VALLEGa (A.), 1996. - «The Agenda 21 of Ocean Geography : The Epistemological Challenge », $28^{\text {th }}$ International Geographical Union Congress, Land, Sea and Human Effort, La Haye, Pays-Bas, non publié.

Van Treeck (P.), Schuhmacher (H.), 1999. - « Mass diving tourism - A new dimensional calls for new management approaches », Marine Pollution Bulletin, 37 (8-12), p. 499-504.

WCPA, 1998. - Economic Values of Protected Areas : Guidelines for Protected Area Managers, Task Force on Economic Benefits of Protected Areas of the World Commission on Protected Areas of IUCN, Gland (Suisse), IUCN, $52 \mathrm{p}$.

Weber (J.), Réveret (J.-P.), 1994. - «Biens communs : les leurres de la privatisation », dans Le Monde Diplomatique, Terre de renaissance, coll. «Savoirs », n², p. 71-73.

Zabala (M.), 1999. - « Recreation in Mediterranean marine parks: limits and perspectives », dans CIESM, Scientific design and monitoring of Mediterranean marine protected areas, Porto Cesareo (Italy), 23-26 October 1999, CIESM Workshop Series n 8, Édition Frédéric Briand, Monaco, p. 51-52.

Cet article a été reçu le 5 février 2007 et définitivement accepté le 28 mai 2007. 